\title{
Salud mental en niños y adolescentes: un estudio exploratorio basado en la atención plena compasiva
}

\author{
Mental health in children and adolescents: \\ An exploratory study based in compassionate mindfulness
}

\author{
Tanya María Fragoso y Ana Moreno Coutiño
}

Universidad Nacional Autónoma de México ${ }^{1}$

Autor para correspondencia: Tanya María Fragoso,tabou_kire@hotmail.com.

\section{RESUMEN}

\begin{abstract}
Se está presenciando en la actualidad una integración masiva de las prácticas meditativas orientales con la teoría y la práctica de la psicología y la psicoterapia. La meditación es utilizada como un instrumento para aliviar el sufrimiento por estrés, tan arraigado en la naturaleza humana. La práctica de la atención plena ha reportado grandes beneficios en distintos padecimientos psíquicos y físicos, ayudando a crear estados de ánimo positivos y promoviendo las actitudes de aproximación, afrontamiento y equilibrio emocional, en lugar de la evitación. Las personas que meditan con regularidad muestran una disposición afectiva positiva, así como una mayor habilidad de afrontamiento de los estados de ánimo negativos. Así, el presente estudio empírico, de carácter cualitativo y cuantitativo, con un diseño preexperimental con un solo grupo tuvo como objetivo conocer el efecto de una intervención basada en la atención plena compasiva en la salud mental de niños y adolescentes de entre 6 y 15 años. Para este propósito, se compararon las evaluaciones previa y posterior a la impartición de un taller. Para el análisis estadístico se llevó a cabo una prueba paramétrica de comparación de dos muestras relacionadas. A lo largo de las sesiones, se pudo observar en los participantes un aumento de la empatía y la compasión hacia sus compañeros. Los principales hallazgos de este estudio fueron las mejorías estadísticamente significativas registradas en la disminución de las alteraciones afectivas, así como de la sintomatología ansiosa. Además, se apreció una tendencia hacia la mejoría en los problemas somáticos, la conducta negativista, el trastorno por déficit de atención e hiperactividad y otros problemas de conducta.
\end{abstract}

Palabras clave: Niños; Adolescentes; Atención plena; Compasión; Meditación.

\begin{abstract}
Currently, there is a massive integration of eastern meditation practices in the theory and practice of psychology and psychotherapy. Meditation is used as an instrument to diminish suffering, so ingrained in human nature. In this context, the practice of Mindfulness has reported great benefits in both mental and physical illnesses. This practice leads to positive states of mind promoting a better approach to them, developing better coping strategies, and promoting emotional balance instead of avoidance. People who meditate regularly develop a positive affective disposition and a better ability to cope with negative mood states. The present study constitutes an empirical investigation of qualitative and quantitative nature which aimed to evaluate the effect of a compassionate mindfulness intervention on the mental health of children and adolescents between 6 and 15 years old.
\end{abstract}

\footnotetext{
${ }^{1}$ Facultad de Psicología, Edificio C, Cubículo 230, Av. Universidad 3004, Col. Copilco Universidad, Coyoacán, Ciudad de México, México, tel. (55)53-70-73-643, correos electrónicos: tabou_kire@hotmail.com y moca99_99@yahoo.com.
} 
The study assessed participants before and after a workshop through a pre-experimental design with a single group. Data analysis used a parametric test to compare two related samples of students enrolled in the meditation workshop in a non-profit organization. Throughout the sessions, it was possible to observe how participants gradually integrated the concepts and techniques taught. Some participants showed increased empathy and compassion toward their peers and desired to help others understand and continue learning. Some participants reported sharing the workshop experiences at home, teaching their parents or siblings to meditate with the mind jar exercise, and the attention to the breathing technique. Results point toward alternative ways to deal with afflictive emotions and the concomitant overflowing reactions.

Keywords: Children; Teenagers; Mindfulness; Compassion; Meditation.

Recibido: $17 / 06 / 2020$

Aceptado: 01/10/2020

\section{ANTECEDENTES}

\section{Terapias conductuales de tercera generación}

$\mathrm{L}$ as terapias de la conducta de tercera generación surgieron en la década de 1990, aunque no fue sino hasta 2004 que fueron consideradas plenamente. Su surgimiento se debió a las limitaciones halladas en la terapia cognitivo-conductual, no obstante haber hecho importantes contribuciones en el área clínica (Pérez, 2006).

Las aportaciones destacadas están relacionadas a un replanteamiento contextual de la psicología clínica, que incluye la psicopatología, la evaluación y el tratamiento; tal replanteamiento contextual es importante, ya que el modelo médico en ocasiones es adoptado de manera inadvertida y hasta cierto punto ingenua (Dougher y Hayes, 2000). Otras aportaciones importantes se encuentran en el tratamiento, que tiene como principio terapéutico "el abandono de la lucha contra los síntomas y, en su lugar, la reorientación de la vida" (Moreno, 2012).

Las principales terapias de tercera generación que existen en la actualidad son la psicoterapia analítica funcional, la terapia de aceptación y com- promiso (ACT), la de conducta dialéctica (DBT), la terapia conductual integrada de pareja, la de activación conductual, la de reducción de estrés basada en atención plena (REBAP, MBSR) y la terapia cognitiva basada en estar atento para el trastorno de ansiedad generalizada (MBCT) (Moreno, 2012). Estas terapias no ponen demasiado énfasis en los síntomas del sujeto; antes bien, pretenden modificar su experiencia subjetiva y la conciencia de ella, de tal modo que los síntomas le resulten al sujeto exagerados u obsoletos (Finucane y Mercer, 2006). De manera particular, las terapias basadas en la atención plena han sido evaluadas en diversas poblaciones con distintos trastornos del estado de ánimo.

Mindfulness es la acepción inglesa de los conceptos en español "atención plena", "presencia plena" o "conciencia plena". Con ella, se trata de prestar atención intencionalmente a la experiencia propia, de momento a momento, aceptándola sin juzgarla, y desarrollando con ello una perspectiva nueva acerca de los pensamientos y sentimientos, por los que se les reconoce como eventos mentales y no como aspectos estables del ser o reflejos exactos de la realidad (Kabat-Zin, 1990).

\section{Evidencias de la atención plena}

Los avances en los métodos para estudiar el cerebro humano, así como las técnicas de neuroimagen y procesamiento computarizado de las señales electroencefalográficas, han permitido el estudio de los procesos neurobiológicos con un alto nivel de precisión (Moreno, 2008). Gracias a esto, ya es posible saber lo que sucede en el cerebro humano cuando se practica regularmente la atención plena. Tal práctica ha resultado efectiva en lo que respecta al equilibrio emocional debido a que ayuda a crear estados de ánimo positivos y promover las actitudes de aproximación y afrontamiento en lugar de las de evitación, utilizadas comúnmente en las terapias de primera y segunda generación.

Los efectos que tiene la atención plena en la actividad cerebral se han relacionado con el estado de ánimo del individuo, llamándolo disposición afectiva. Davidson et al. (2003) llegaron a la conclusión de que las personas que meditan con regularidad, al ser comparadas con las que no lo 
hacen, manifiestan un incremento mayor de la activación cerebral izquierda en las zonas cerebrales anteriores y medias, las cuales están relacionadas con una disposición afectiva positiva (Simón, 2007). Dichos resultados deben interpretarse con base en las investigaciones que afirman la relación entre los estados afectivos positivos y el predominio de la activación cerebral anterior izquierda, y en el caso de los estados emocionales negativos, con el de la activación cerebral anterior derecha (Davidson et al., 2003).

La activación cerebral anterior izquierda se ha asociado asimismo con una mayor habilidad de afrontamiento ante los estados de ánimo negativos, así como con la disminución de este tipo de afectos, demostrado en los estudios de Sutton y Davidson (1997), Davidson e Irwin, (1999), Tomarken et al. (1990), entre otros (cf. Simón, 2007).

Otro descubrimiento ha sido la influencia de la práctica de la atención plena en los mecanismos inmunitarios, gracias al cual se concluyó que la meditación potencia la producción de anticuerpos debido a que uno de los mecanismos por los que el estrés ejerce su mecanismo negativo sobre la salud es a través del aumento del cortisol y de sus consiguientes efectos inmunodepresores. Por ello, es probable que los efectos positivos de la práctica de la meditación sobre la inmunidad estén causados por la disminución de la secreción de cortisol en respuesta al estrés (Simon, 2007).

Por otro lado, en el decenio de 1990 un grupo de investigadores italianos (Fadiga, Gallese y Fogassi, 1996; Rizzolatti y Arbib, 1998) hallaron que en la corteza premotora de monos se activaban cierto tipo de neuronas al realizar una acción, pero también cuando un animal observaba a otros ejecutando la misma tarea, a las que se denominó "neuronas espejo". Gracias a estas redes neuronales en espejo es que se pueden entender mejor las emociones del otro y la simulación posterior. La simple observación de las acciones de otros seres vivos permite inferir, además de sus intenciones, los estados emocionales que las sustentan (Moreno, 2008).

Los mecanismos que generalmente se encargan de sustentar las experiencias de sincronización y resonancia con otras personas (empatía y compasión) en la práctica de la atención plena, se vuelven además sobre el individuo para desarrollar una relación de amor sin temor a la propia experiencia. Siegel (2001) afirma que la atención plena promueve las relaciones sanas a partir de la mayor empatía aumentada, el equilibrio emocional, la flexibilidad de respuestas y la predisposición a la aproximación. Y así, gracias a la práctica se generan valores como la empatía, la comprensión, el amor y la compasión en nuestra propia experiencia. Esta es la hipótesis de autoempatía o de sincronización interna a la que llegó Siegel en 2007 (cf. Simón, 2007).

Se ha comprobado también que las áreas cerebrales activadas por la empatía y la compasión son muy distintas; en la primera, al nutrir el dolor del otro, se experimentan también sus emociones aflictivas y la ya citada resonancia empática; cuando esto se repite muchas veces puede producir dolor, agotamiento emocional y un sentimiento de desamparo, como es el caso del burnout, también conocido como "síndrome del profesional quemado"; en muchas ocasiones, aun cuando las emociones son positivas, se pueden generar actitudes sectarias mediante la empatía. Pero cuando se medita sobre la compasión y el amor altruista se está ante la posibilidad de desarrollar emociones positivas, lo que refuerza el ánimo, el equilibrio interior y la determinación, valiente y afectuosa, de ayudar a los que sufren. En esencia, el amor y la compasión no generan fatiga ni desgaste, sino que, por el contrario, ayudan a superarlos y a repararlos (Ricard, 2014).

La terapia cognitiva basada en la atención plena ha demostrado ser eficaz en el tratamiento de pacientes con depresión crónica (Teasdale et al., 2000), trastornos de personalidad (Linehan et al., 2006; Van den Bosch, Koeter, Stijnen, Verheul y Van Den Brink, 2005), abuso de sustancias (Davis, Fleming, Bonus y Baker, 2007; Van den Bosch et al., 2005), cáncer (Carlson, Speca, Patel y Goodey, 2004; Tacón et al., 2004), dolor crónico (Plews-Ogan, Owens, Goodman, Wolfe y Schorling, 2005; Sagula y Rice, 2004), entre otros. De igual manera, distintos estudios controlados aleatorizados han comprobado el efecto positivo de la práctica de la mindfulness based stress reduction (MBSR) en poblaciones no clínicas (Pérez y Bote1la, 2006). 


\section{Aplicación de la atención plena en la población infantil y adolescente}

En las últimas décadas, la investigación sobre la atención plena se ha centrado principalmente en poblaciones adultas; sin embargo, también se han desarrollado intervenciones que han hecho un esfuerzo por adaptar los programas a la población infantil y adolescente (Black y Fernando, 2014; Hennelly, 2011; Lau y Hue, 2011), como el Mindfulness-based Kindness Curriculum (KC) (Flook, Goldberg, Pinger y Davidson, 2014), el Mindful School (MS) (Black y Fernando, 2013), el programa MYmind (Haydick et al., 2013), el Mind UP integrado en un programa de aprendizaje social y emocional SEL (Schonert-Reichl et al., 2015), el programa Inner Kids (Kaiser, 2010; $c f$. Casado y Caballo 2015), el Mindfulness in Schools Project's MisP (Hennelly, 2011; Huppert y Johnsosn 2010), el programa de Educación en Mindfulness ME (Schonbert-Reichl y Lawlor, 2015) y el Master Mind (Parker, Kupersmidt, Mathis, Scull y Sims, 2014).

Por otro lado, en la literatura internacional se hallan diversos estudios epidemiológicos que señalan que los trastornos con mayor prevalencia durante la infancia y la adolescencia son los de ansiedad (Costello, Mustillo, Erkanli, Keeler y Angold, 2003; Essau, Conradt y Petermann, 2000; Muris, Mayer, Bartelds, Tierney y Bogie, 2001), en que las tasas van de $2.6 \%$ a $41.2 \%$. Se ha documentado que dichos trastornos pueden comenzar en la niñez (hasta 75\%), hacia los 11 años de edad, siguiendo un curso de deterioro en la adolescencia y la adultez, y que se vinculan con problemas tales como el bajo rendimiento escolar, deficiencias en la interacción social y conductas de aislamiento. Su comorbilidad con la depresión se calcula entre 30 y $60 \%$ (Essau, Conradt y Petermann, 2000), lo que constituye uno de los problemas de salud pública más importantes en todo el mundo y que deja muy clara la necesidad de generar intervenciones efectivas para el manejo de la sintomatología ansiosa desde esa etapa de la vida.

En México, las investigaciones desarrolladas en torno a las intervenciones basadas en la atención plena son aún incipientes, y más aún en me- nores de edad, a pesar de la gran relevancia que las mismas han alcanzado en muchos otros países como alternativa psicológica para el tratamiento de pacientes con trastornos relacionados al estrés, la ansiedad y la depresión en los últimos años. Por tal motivo, resulta necesario evaluar este tipo de intervenciones en los niños y adolescentes mexicanos, por lo que la presente investigación, con un diseño preexperimental, con evaluación pre-post, sin grupo de comparación (Campbell y Stanley, 2005), se planteó con la intención de conocer el efecto de una intervención basada en atención plena compasiva (APC en lo sucesivo) sobre los problemas físicos, escolares, sociales y emocionales de niños y adolescentes, comparando las evaluaciones previa y posterior a la intervención, consistente en un taller basado en la atención plena compasiva que contribuya a la disminución de aquellos entre la mencionada población, y asimismo evaluar si la intervención basada en APC disminuye tal problemática de una forma estadísticamente significativa.

\section{MÉTODO}

\section{Participantes}

La selección de los participantes fue intencional y se realizó con los alumnos que se inscribieron al "Taller de atención plena compasiva para niños y adolescentes" en la organización privada Integritat, dedicada al apoyo a niños en situación de calle o de escasos recursos. A este efecto, se organizó una sesión informativa de los talleres que se impartirían en las instalaciones de la institución. Los padres de los niños que asistieron $(\mathrm{n}=30)$ fueron invitados a que sus hijos participaran en el taller. Las características de los participantes que se reportan en este estudio son las que se muestran en la Tabla 1.

Para garantizar los resultados válidos y confiables de la intervención, los participantes debían cumplir con los siguientes criterios de inclusión: tener entre 6 y 15 años de edad, estar inscritos en el "Taller de atención plena compasiva" en Integritat, haber recibido el consentimiento informado 
Tabla 1. Datos descriptivos de los participantes.

\begin{tabular}{|c|c|c|c|c|c|}
\hline Sexo & Cantidad & $\begin{array}{c}\text { Edad } \\
\text { en años }\end{array}$ & $\%$ & Escolaridad & $\%$ \\
\hline Mujeres & 9 & $6-9$ & 60 & $1^{\circ}-20$ Primaria & 26.66 \\
\hline Hombres & 6 & $10-12$ & 20 & 30 - 40 Primaria & 40.00 \\
\hline Total & 15 & $13-15$ & 20 & $10-3^{\circ}$ Secundaria & 33.33 \\
\hline & & $\mathrm{n}=15$ & 100 & $\mathrm{n}=15$ & 100.00 \\
\hline
\end{tabular}

de sus padres o tutores, establecer el compromiso para participar en todas las sesiones del taller y responder las evaluaciones previas y posteriores a la intervención. Se excluyó a todos aquellos que no desearan participar en el mismo, abandonaran el taller o incumplieran cualquiera de las dos evaluaciones requeridas.

\section{Variable independiente}

Taller basado en la APC para niños y adolescentes de 6 a 15 años.

Definición conceptual. La atención plena compasiva se refiere a la conciencia que surge a través de prestar atención propositivamente al momento presente, sin juzgar la experiencia y generando acciones motivadas en la aspiración de liberarse a uno mismo y a los demás del sufrimiento.

Definición operacional. El taller basado en la atención plena compasiva para niños y adolescentes de 6 a 15 años es de modalidad grupal, que se imparte en ocho sesiones de 50 minutos cada una, en el cual los participantes aprenden, mediante dinámicas y diversas técnicas de meditación, cómo mantener su atención en el presente, manejar sus emociones, cultivar la atención, la concentración, las emociones positivas y la compasión.

\section{Variables dependientes}

\section{Salud mental}

Definición conceptual. La salud mental abarca una amplia gama de actividades directa o indirectamente relacionadas con el componente de bienestar mental incluido en la siguiente definición: "Un estado de completo bienestar físico, mental y social, y no solamente la ausencia de afecciones o enfermedades" (Organización Mundial de la Salud, 2019).

Definición operacional. Puntuación obtenida en el Cuestionario de Comportamiento Infantil

Instituto de Investigaciones Psicológicas - Universidad Veracruzana de 6 a 18 años (Child Behavior Checklist, $C B C L$ 6-18), que es una de las listas de síntomas más usadas para evaluar la psicopatología más común en dicha población y presente en los últimos seis meses ( $c f$. Verhulst, Koot y Van der Ende, 1992). Las dimensiones que explora el CBCL incluyen las escalas orientadas a los problemas afectivos, de ansiedad, de conducta, somáticos y relacionados con el TDAH.

\section{Instrumentos}

Cuestionario de Comportamiento Infantil de 6 a 18 años (CBCL 6-18) (Achenbach y Rescorla, 2000).

Esta escala se compone de seis subescalas basadas en el DSM-V que evalúan los niveles de problemas somáticos, afectivos, de ansiedad, de conducta, TDAH, trastorno negativista desafiante y está validada en población mexicana por Albores et al. (2007). La consistencia interna de la escala se examinó mediante el coeficiente alfa de Cronbach para todas las subescalas, considerándose buenos los valores de 0.61 a 0.79 , y excelentes los iguales o superiores a 0.80 .

\section{Procedimiento}

La asignación de los participantes se realizó de un modo no aleatorio con el grupo de alumnos inscritos en el Taller de Meditación de la Asociación Integritat, por lo que el estudio fue de carácter exploratorio.

\section{Sesión informativa}

La sesión informativa tuvo una duración de $30 \mathrm{mi}-$ nutos y se llevó a cabo grupalmente una semana antes de empezar la intervención. En ella se explicó a los 25 interesados el objetivo del taller, quienes fueron registrados, y después se les entregó 
una ficha de identificación, junto con el Cuestionario de Comportamiento Infantil (CBCL) para ser contestados en casa.

\section{Intervención}

El taller se aplicó en modalidad grupal, en el cual, mediante dinámicas y diversas técnicas de meditación, los niños aprenden a manejar sus emocio- nes, cultivar la concentración y la atención plena compasiva, promoviendo el pensamiento reflexivo. El taller consta de ocho sesiones, cada una con una duración de 50 minutos una vez por semana. Las sesiones fueron impartidas en un aula de las instalaciones de Integritat por dos psicólogas previamente capacitadas en el modelo de atención plena compasiva.

El proceso del estudio fue el siguiente:

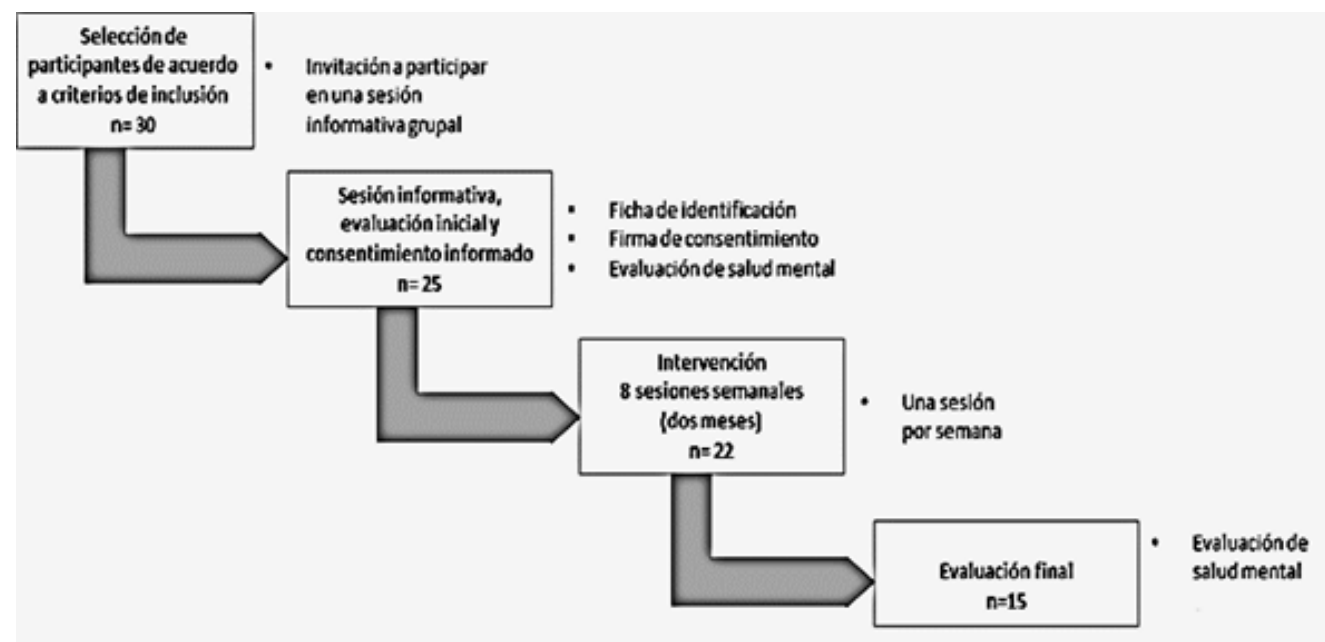

Las sesiones tuvieron los siguientes objetivos:

Sesión 1. Reconocer las propias virtudes para así fortalecerlas y aprender a reconocerlas en los demás mediante la reflexión y de una actividad llamada "Flor de loto".

Sesión 2. Conocer la naturaleza de la mente. Identificar cómo es que surgen en ella todos los eventos mentales (emociones, sensaciones, percepciones, pensamientos, imágenes, recuerdos, etc.), cómo se manifiestan y las consecuencias de familiarizarse con ellos.

Sesión 3. Reflexionar sobre los beneficios de entrenar la mente para que esté serena. Se enseñará la técnica de meditación con apoyo en un objeto, guiando para experimentar la diferencia que existe en la percepción de las cosas y situaciones cuando la mente está en paz y no agitada.

Sesión 4. Desarrollar en los participantes la conciencia corporal y ayudarlos a desarrollar su atención y concentración.

Sesión 5. A través de un cuento que relata una escena de la infancia del Buda Siddhartha Gauta- ma, invitar a los participantes a reflexionar y meditar sobre el significado de la compasión y la interdependencia.

Sesión 6. Analizar cómo todas las acciones y situaciones tienen causas y consecuencias

Sesión 7. Practicar la meditación en movimiento través de un paseo lento y silencioso, llevando toda la atención a los diferentes momentos de la caminata e intentando llevarla a cabo lo más lentamente posible estando consciente de cada movimiento.

Sesión 8. Realizar una meditación guiada que ayuda para la comprensión de la interdependencia que existe entre todos los fenómenos. Para esta última sesión se sugirió a los participantes que se pusieran de acuerdo entre ellos para repetir algunas de las actividades cumplidas a lo largo del taller.

En el transcurso de las sesiones se les enseñó a realizar meditaciones de manera gradual a lo largo de la semana, dejándoles como tarea ciertas actividades para hacer en casa, con el fin de que fueran familiarizándose con las técnicas y refor- 
zando los conocimientos. En un primer momento, el grupo quedó conformado por veinticinco niños, aunque después tres de ellos se dieron de baja por diversas razones durante el taller.

\section{Evaluaciones postintervención}

Tras concluir la última sesión de la intervención, se les pidió a los padres que les ayudaran a los niños y adolescentes a contestar el Cuestionario de Comportamiento Infantil. Solo quince de los participantes entregaron la evaluación completa, por lo que para el análisis estadístico del presente estudio se consideraron únicamente a estos.

\section{Análisis estadístico}

De manera inicial se efectuó una descripción general de la muestra a través de medidas de tendencia central y de dispersión. Luego, se analizaron las diferencias entre las dos evaluaciones sobre cada una de las variables dependientes aplicándose la $t$ de Student para comparar las medias de la evaluación previa al taller y la posterior.

\section{RESULTADOS}

En la Gráfica 1 se muestran las medias de los puntajes del CBCL obtenidos en la evaluación previa $(\overline{\mathrm{x}}=16.91)$ y posterior al taller $(\overline{\mathrm{x}}=14.14) . \mathrm{Al}$ analizar los datos mediante la prueba $t$ de Student se registró una disminución significativa tanto de los problemas afectivos $(t[10]=2.622, p<0.05)$ como en los de ansiedad $(t[10]=2.292, p<0.05)$. Los puntajes obtenidos en estas dos variables antes y después del taller se encuentran en un rango normal; es decir, los síntomas mostrados por los niños eran los esperados para su edad, por lo que no correspondían a un nivel clínico.

Gráfica 1. Puntajes obtenidos antes y después del taller. El inciso $(*)$ indica los problemas en los que se registró un cambio significativo: problemas afectivos y problemas de ansiedad.

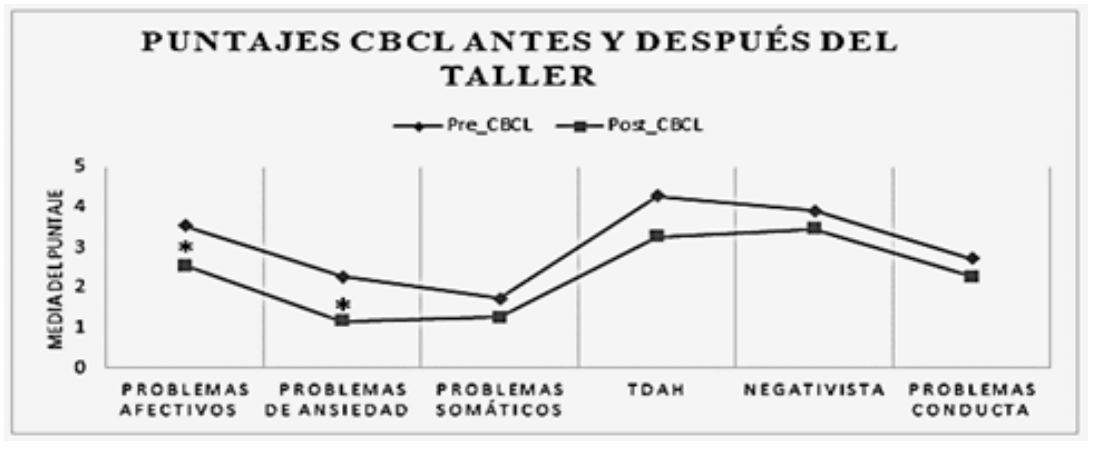

Con tales resultados es posible concluir que se confirmó la hipótesis de que existen diferencias estadísticamente significativas entre la evaluación basal y la evaluación realizada al finalizar el Taller basado en Atención Plena Compasiva para niños y adolescentes de 6 a 15 años sobre su salud mental.

\section{DISCUSIÓN}

El principal hallazgo de este trabajo fue la mejoría estadísticamente significativa de los proble- mas afectivos y los de ansiedad registrada en los puntajes previos y posteriores al taller. Al mismo tiempo, se observó una tendencia a la mejoría, aunque no estadísticamente significativa, relativa a los problemas de conducta, somáticos, negativistas $\mathrm{y}$ asociados al TDAH.

Una posible explicación de por qué no se registró una diferencia significativa al evaluar a los participantes es que sus edades eran muy diversas, por lo que las dinámicas y actividades no tuvieron el mismo efecto en todos ellos. También se puede inferir que el número de sesiones no fue suficiente 
para lograr desarrollar un cambio evidente, y que los beneficios de la intervención no pudieron ser correctamente discriminados mediante la prueba utilizada.

Lo anterior resulta particularmente relevante debido a que los problemas asociados al estado de ánimo se encuentran estrechamente vinculados al desarrollo de diversos padecimientos que surgen durante la niñez y la adolescencia, al igual que la aparición de ciertos trastornos de la personalidad en la edad adulta. Por ejemplo, se ha visto que la impulsividad y la falta de autocontrol presentes en la infancia pueden generar conductas antisociales o delictivas en la edad adulta; asimismo, a la reactividad psicofisiológica e hipersensibilidad al estrés en la infancia se les ha relacionado con la aparición de trastornos afectivos en la adultez. Ofrecer a los niños y jóvenes espacios en los que aprendan a manejar sus emociones resulta, pues, una tarea insoslayable para la prevención de tal tipo de trastornos.

A manera de evaluación cualitativa general de la retroalimentación brindada por los participantes del taller, resulta importante mencionar que tanto los niños como los padres de familia se acercaron a los terapeutas durante y al finalizar el mismo para expresar su interés por las técnicas aprendidas y agradecer el beneficio que les habían brindado en sus diversas experiencias.

Al comparar los resultados de esta investigación con los de estudios previos sobre atención plena compasiva, se puede observar una similitud en el decremento significativo del malestar y la regulación emocional de los participantes (Casado y Caballo, 2015; Crescentini, Capurso, Furlan y Fabbro, 2016, entre otros). La práctica regular de estas actividades a lo largo de nueve semanas parece aumentar la sensación de bienestar referida por los participantes.

Los resultados de la puesta en práctica de los programas de atención plena en entornos escolares muestran que la corta duración de los talleres, así como la brevedad en los tiempos dedicados a la práctica y las reflexiones, impiden que los par- ticipantes obtengan resultados a largo plazo; por lo tanto, podrían considerarse dichos programas como una herramienta introductoria para aquellos que, tras conocer sus bases, deseen practicarla y ahondar en ella más adelante (Burnett, Cullen y O’Neill, 2011). Conseguir un estado de bienestar es un trabajo complejo que requiere de disciplina y constancia en la práctica, por lo que un curso introductorio de algunas semanas es insuficiente (Burnett, 2010). Por lo tanto, se propone perfeccionar dichos programas educando y fomentando la duración de la práctica en el tiempo, así como gestionar con las instituciones los espacios para la supervisión y evaluación de la práctica a largo plazo. Para este propósito resulta imprescindible la formación adecuada del profesorado y de las autoridades educativas para promover y facilitar la práctica de manera cotidiana en las instituciones (Greenberg y Harris, 2012).

Para lograr tener sociedades más sanas, resulta fundamental tener en mente que la implementación de programas de atención plena en las escuelas proporciona a los alumnos una manera de combatir el estrés en un mundo complejo, teniendo como objetivo principal su desarrollo personal y la creación de una sociedad con mayor bienestar (López, 2016).

Por último, sería deseable evaluar este taller en poblaciones delimitadas por grupos de edad homogéneos a fin de capturar la atención de los participantes con actividades mejor adaptadas a sus edades e intereses, favoreciendo así la obtención de los objetivos propuestos. En lo que respecta a las evaluaciones, resultaría importante utilizar instrumentos menos extensos para facilitar su entrega en tiempo y forma.

A pesar de las limitaciones expuestas, los resultados positivos obtenidos sugieren que el Taller de Atención Plena Compasiva puede ser apropiado para mejorar la regulación emocional de los alumnos, así como para ayudarles a manejar la ansiedad y las situaciones difíciles que viven a diario, para que así las nuevas generaciones sean más sanas y felices.

Citación: Fragoso, T.M. y Moreno C., A. (2021). Salud mental en niños y adolescentes: un estudio exploratorio basado en la atención plena compasiva. Psicología y Salud, 32(1), 105-114. https:// Doi.org/10.25009/pys.v32i1.2717. 


\section{REFERENCIAS}

Achenbach, T. y Rescorla, L. (2000). Manual for the ASEBA school-age forms and profiles. Burlington, VT: University of Vermont.

Albores, L., Lara, C., Esperón, C., Cárdenas, J., Pérez, A. y Villanueva, G. (2007). Validez y confiabilidad del CBCL/6-18. Actas Españolas de Psiquiatría, 35(6), 393-399.

Black, D.S. y Fernando, R. (2014). Mindfulness training and classroom behavior among lower-income and ethnic minory. Journal of Child and Family Studies, 23(7), 1242-1246. Doi: 10.1007/s10826-013-9784-4.

Burnett, R. (2010). Mindfulness in schools, learning lessons from the adults - secular and Buddhist. Buddhist Studies Review, 28(1), 79-120.

Burnett, R., Cullen, C. y O’Neill, C. (2011). Mindfulness in Schools Projects. National Library of Medicine, 39(4), 409-414.

Campbell, D.T. y Stanley, J.C. (2005). Diseños experimentales y cuasiexperimentales en la investigación social. Buenos Aires: Amorrortu Editores.

Carlson, L., Speca, M., Patel, K.D. y Goodey, E. (2004). Mindfulness-based stress reduction to quality of life, mood, and symptoms of stress and levels of cortisol, dehydroepiandrosterone sulfate (DHEAS) and melatonin in breast and prostate cancer outpatients. Psychoneuroendocrinology, 29(4), 448-474.

Casado S., P. y Caballo E., C. (2015). Mindfulness en la infancia y la adolescencia. Salamanca (España): Universidad de Salamanca.

Costello, E.J., Mustillo, S., Erkanli, A., Keeler, G. y Angold, A. (2003). Prevalence and development of psychiatric disorders in childhood and adolescence. Archives of General Psychiatry, 60(8), 37-44.

Crescentini, C., Capurso, V., Furlan, S. y Fabbro, F. (2016). Mindfulness-oriented meditation for primary school children: effects on attention and psychological well-being. Frontiers in Psychology, 7(7), 805.

Davidson, R. Kabat-Zinn, J., Schumacher, Rosenkranz, M., Muller, D., Santorelli, S., Urbanowski, F., Harrington, A., Bonus, K. y Sheridan, J. (2003). Alterations in brain and immune function produced by mindfulness meditation. Psychosomatic Medicine, 65(4), 564-570.

Davis, J., Fleming, M., Bonus, K. y Baker, T.B. (2007). A pilot study on mindfulness based stress reduction for smokers. $B M C$ Complementary and Alternative Medicine, 25, 7:2. Doi: 10.1186/1472-6882-7-2.

Dougher, M.J. y Hayes, S.C. (2000). Clinical behavior analysis. Reno, NV: Context Press.

Essau, C.A., Conradt, J. y Petermann, F. (2000). Frequency, comorbidity, and psychosocial impairment of anxiety disorders in German adolescents. Journal of Anxiety Disorders, 14(3), 263-279. Doi: 10.1016/S0887-6185(99)00039-0.

Fadiga, L., Gallese, V. y Fogassi, L. (1996). Premotor cortex and the recognition of motor actions. Cognitive \& BrainResearch, $3(2), 131-141$.

Finucane, A. y Mercer, S.W. (2006). An exploratory mixed methods study of the acceptability and effectiveness of mindfulness-based cognitive therapy for patients with active depression and anxiety in primary care. BMC Psychiatry, 6(14), 1-14.

Flook, L., Goldberg, S.B., Pinger, L. y Davidson, R.J. (2014). Promoting prosocial behavior and self-regulatory skills in preschool children through a mindfulness-based kindness curriculum. Developmental Psychology, 50(1), 44-51.

Hennelly, S. (2011). The immediate and sustained effect of the mindfulness programmed on adolescents social and emotional well-being and academic functioning. Oxford: Oxford Brookes University.

Huppert, F.A. y Johnson, D.M. (2010). A controlled trial of mindfulness training in schools, the importance of practice for an impact on well-being. The Journal of Positive Psychology, 5(4), 264-274.

Kabat-Zinn, J. (1990). Full catastrophe living. Using the wisdom of your body and mind to face stress, pain, and illness. London: Delta.

Kaiser, G.S. (2010). El niño atento: Mindfulness para ayudar a tu hijo a ser más feliz, amable y compasivo. Bilbao: Desclée de Brouwer.

Lau, N.S. y Hue, M.T. (2011). Preliminary outcomes of a mindfulness-based programme for Hong Kong adolescents in schools: Well-being, stress and depressive symptoms. International Journal of Children's Spirituality, 16(4), 315-330.

Linehan, M.M., Comtois, K.A., Murray, A.M., Brown, M.Z., Gallop, R.J., Heard, H.L., Korsulund, K.E., Tutek, D.A., Reynolds, S.K. y Lindenboim, N. (2006). Two-year randomized controlled trial and follow-up of dialectical behavior therapy vs. therapy by experts for suicidal behavior and borderline personality disorder. Archives of General Psychiatry, 63(7), 757-766.

López H., L. (2016). Técnicas Mindfulness en centros educativos. Desarrollo académico y personal de sus participantes. Revista Española de Orientación y Psicopatología, 27(1), 134-146.

Moreno C., A. (2012). Terapias cognitivo-conductuales de tercera generación (TTG): La atención Plena / Mindfulness. Revista Internacional de Psicología, 12(1), 1-18. Doi: 10.33670/18181023.v12i01.66. 
Moreno, N. (2008). La práctica del mindfulness (Atención plena). Andalucía (España): Centro de Psicología Clínica Nicolás Moreno. Recuperado de: http://www.nicolasmorenopsicologo.com/tratamientos/centro_articulos.htm.

Organización Mundial de la Salud (2019). Constitución de la Organización Mundial de la Salud (Definición de adolescencia). Ginebra: OMS.

Parker, A.E., Kupersmidt, J.B., Mathis, E.T., Scull, T.M., y Sims, C. (2014). The impact of mindfulness education on elementary school students: Evaluation of the Master Mind program. Advances in School Mental Health Promotion, 7(3), 184-204.

Pérez Á., M. (2006). La terapia de conducta de tercera generación. Revista de Psicología y Psicopedagogía, 5(2), 159-172.

Pérez, M.A. y Botella, L. (2006). Consciencia plena (mindfulness) y psicoterapia: concepto, evaluación y aplicaciones clínicas. Revista de Psicoterapia, 17(66/67), 77-120.

Plews-Ogan, M., Owens, J.E., Goodman, M., Wolfe, P. y Schorling, J. (2005). A pilot study evaluating mindfulness-based stress reduction and massage for the management of chronic pain. Journal of General Internal Medicine, 20(12), 1136-1138.

Ricard, M. (2014). El defensa del altruismo. Barcelona: Urano.

Rizzolatti, G. y Arbib, M. (1998). Language within our grasp. Trends in Neurosciences, 21, 188-194. Doi: 10.1016/S01662236(98)01260-0.

Sagula, D. y Rice, K.G. (2004). The effectiveness of Mindfulness training on the grieving process and emotional well-being of chronic pain patients. Journal of Clinical Psychology in Medical Settings, 11(4), 333-341.

Schonert-Reichl, K.A., Oberle, E., Lawlor, M.S., Abbott, D., Thomson, K., Oberlander, T.F. y Diamond, A. (2015). Enhancing cognitive and social-emotional development through a simple to-administer mindfulness-based school program for elementary school children: A randomized controlled trial. Developmental Psychology, 51(1), 52-66.

Siegel, D.J. (2001). Toward an interpersonal neurobiology of the developing mind: attachment relationships, "mindsight," and neural integration. Infant Mental Health Journal, 22(1-2), 67-94.

Siegel, D.J. (2007). The mindful brain. New York: Norton \& Co.

Simón, V. (2007). Mindfulness y neurobiología. Revista de Psicoterapia, 17(66/67), 5-30.

Sutton, S.K. y Davidson, R.J. (1997). Prefrontal brain asymmetry: a biological substrate of the behavioral approach and inhibition systems. Psychological Science, 8(3), 204-210.

Teasdale, J.D., Segal, Z.V., Williams, J.M., Ridgeway, V.A., Soulsby, J.M. y Lau, M.A. (2000). Prevention of relapse/recurrence in major depression by mindfulness-based cognitive therapy. Journal of Consulting and Clinical Psychology, 68(4), 615-623.

Van Den Bosch, L.M.C., Koeter, M.W.J., Stijnen, T., Verheul, R. y Van Den Brink, W. (2005). Sustained efficacy of dialectical behaviour therapy for borderline personality disorder. Behaviour Research and Therapy, 43(9), 1231-1241.

Verhulst, F.C. Koot, H.M. y Van der Ende, J. (1992). Valor predictivo diferencial de los informes de padres y docentes sobre conductas problemáticas de los niños: un estudio longitudinal. Revista de Psicología Infantil Anormal, 22, 531-546.

Instituto de Investigaciones Psicológicas - Universidad Veracruzana

ISSN impreso: 1405-1109
Psicología y Salud, Vol. 32, Núm. 1: 105-114, enero-junio de 2022 https://doi.org/10.25009/pys.v32i1.2717 